\title{
Synthesis of Tin Oxide Nanofibers for Lung Cancer, Diabetes and Kidney Malfunction Detection
}

\author{
Florina Regius ${ }^{1}$, Shrey Jain ${ }^{2}$ \\ ${ }^{1}$ Nantechnology Research Center, SRM University, Chennai, T.N \\ ${ }^{2}$ Noida Institute of Engineering and Technology
}

\begin{abstract}
A highly sensitive exhaled-breath sensor, using tin dioxide (SnO2) fibers assembled from thin, wrinkled SnO2 nanotubes can be developed. These metal-oxide nanofiber-based chemiresistive gas sensors allow for portable real-time breath tests that could be available on smart phones or tablets in the near future. They sensors allow for diagnosing serious diseases such as diabetes or lung cancer quickly and effectively by simply breathing into a small nanofiber breathing sensor, mounted on a phone or other device..The SnO2 fibers, called "micro phase-separations," are generated between polymers and dissolved materials while varying the flow rate of an electrospinning solution feed and then applying a heat treatment.
\end{abstract}

Keywords: nanotubes, nanofiber, chemiresistive, polymers

\section{Introduction}

The analysis of volatile organic compounds in exhaled breath samples represents a new frontier in medical diagnostics because it is a noninvasive and potentially inexpensive way to detect illnesses. Clinical trials with spectrometry and spectroscopy techniques, the standard volatile-compound detection methods, have shown the potential for diagnosing illnesses including cancer, multiple sclerosis, Parkinson's disease, tuberculosis, diabetes, and more via breath tests. Unfortunately, this approach requires expensive equipment and high levels of expertise to operate the necessary instruments, and the tests must be done quickly and use preconcentration techniques, all of which impede its adoption. Sensing matrices based on nanomaterials are likely to become a clinical and laboratory diagnostic tool because they are significantly smaller, easierto-use, and less expensive than spectrometry or spectroscopy. An ideal nanomaterial-based sensor for breath testing should be sensitive at very low concentrations of volatile organic compounds, even in the presence of environmental or physiological confounding factors. It should also respond rapidly and proportionately to small changes in concentration and provide a consistent output that is specific to a given volatile organic compound. When not in contact with the volatile organic compounds, the sensor should quickly return to its baseline state or be simple and inexpensive enough to be disposable. Several reviews have focused on the methodological, biochemical, and clinical aspects of breath analysis in attempts to bring breath testing closer to practice for comprehensive disease detection. This Account pays particular attention to the technological gaps and confounding factors that impede nanomaterial-sensorbased breath testing, in the hope of directing future research and development efforts towards the best possible approaches to overcome these obstacles. We discuss breath testing as a complex process involving numerous steps, each of which has several possible technological alternatives with advantages and drawbacks that might affect the performance of the nanomaterial-based sensors in a breath-testing system. With this in mind, we discuss how to choose nanomaterialbased sensors, considering the profile of the targeted breath markers and the possible limitations of the approach, and how to design the surrounding breath-testing setup. We also discuss how to tailor the dynamic range and selectivity of the applied sensors to detect the disease-related volatile organic compounds of interest. Finally, we describe approaches to overcome other obstacles by improving the sensing elements and the supporting techniques such as preconcentration and dehumidification. Detecting a disease through the smell print of a person's breath, via a signature of volatile organic compounds (VOCs), has been long recognized as having great potential as a rapid and noninvasive method for widespread screening and disease diagnosis. Clinical trials have shown the possibility of using breath for detecting serious illnesses, such as different types of cancer, multiple sclerosis . Sensors for Breath Testing Parkinson's and Alzheimer's disease, tuberculosis, diabetes, and chronic kidney disease. Several reviews have covered important biochemical, clinical, and methodological aspects of breath testing, while other reviews have highlighted considerable advances in gas and VOC sensing technologies. Important milestones have been reached in the field of breath analysis. Nevertheless, only few breath tests are currently practiced in the clinic. This situation is primarily a consequence of the technological difficulties in the detection of trace amounts of disease-related VOCs within a complex exhaled breath sample. An additional reason for this situation is that the wide variety of implemented techniques causes a major standardization problem in the field. Part of these methods is still premature and, therefore, confined to research. The other part of the methods include established analytical approaches, such as mass spectrometry, but these tools suffer from high costs, complexity, and require trained personnel for their operation. In recent years, special attention has been given to methods incorporating nanomaterial based VOC/gas sensors (NMVSs) because they would enable the development of highly sensitive, rapidly responsive, and yet cheap detection systems. These virtues could be attributed to the fact that nanoscale dimensions are associated with unique and controllable physical, chemical, and optical properties as well as with low cost fabrications. For instance, the dynamic range as 


\section{International Journal of Science and Research (IJSR) \\ ISSN (Online): 2319-7064}

Index Copernicus Value (2013): 6.14 | Impact Factor (2014): 5.611

well as the selectivity of the NMVSs can be tailored to accurately detect specific breath VOCs of a given disease.

\section{Experimental Method}

Synthesis of $\mathrm{SnO}_{2}$ Nanofibers. $\mathrm{SnO}_{2}$ nanofibers were electrospun from solutions containing stannous chloride $\left(\mathrm{SnCl}_{2} \cdot 2 \mathrm{H}_{2} \mathrm{O}\right)$, polyvinyl pyrrolidone (PVP, molecular weight $\left.\left(\mathrm{M}_{\mathrm{w}}\right)=1300000\right)$, polyacrylonitrile $\left(\mathrm{PAN}, \mathrm{M}_{\mathrm{w}}=\right.$ 150 000) and dimethylformamide (DMF). Two precursor solutions were prepared as follows. Solution A. A $0.02 \mathrm{M}$ sample of stannous chloride was dissolved in $20 \mathrm{~mL}$ of DMF mixed and stirred for $6 \mathrm{~h}$. Subsequently, $6 \mathrm{~g}$ of PVP was dissolved in the solution and continuously stirred for $12 \mathrm{~h}$ using vigorous magnetic stirring. Solution B. Here, $1 \mathrm{~g}$ of PAN was dissolved in $10 \mathrm{~mL}$ of DMF and kept at $80{ }^{\circ} \mathrm{C}$ for $12 \mathrm{~h}$ and cooled down to the room temperature for $12 \mathrm{~h}$. Consequently, $4 \mathrm{~mL}$ of solution A was mixed with 1,2, and $3 \mathrm{~mL}$ of solution $\mathrm{B}$ which are denoted as PAN 1, PAN 2, and PAN 3, respectively. All these solutions were then magnetically stirred for $3 \mathrm{~h}$ at speed of $1000 \mathrm{rpm}$ for PAN 1 and PAN 2, and $1500 \mathrm{rpm}$ for PAN 3, and separately electrospun on quartz substrates attached on rotating electrode under an applied voltage of $20 \mathrm{kV}$, by using a syringe that was kept at a distance of $15 \mathrm{~cm}$ from the quartz substrate. The electrospun were conducted for $40 \mathrm{~min}$ for each sample. The apparatus utilized for electrospinning is depicted in Figure 1a. The flow rate of the solutions was kept at $0.4 \mathrm{~mL} / \mathrm{h}$ during the electrospinning process. The aselectrospun samples were calcined for $4 \mathrm{~h}$ at $500{ }^{\circ} \mathrm{C}$ in air at a heating ramping up of $5{ }^{\circ} \mathrm{C} / \mathrm{min}$. During heat treatment, PAN and PVP were decomposed and the $\mathrm{SnCl}_{2} \cdot 2 \mathrm{H}_{2} \mathrm{O}$ precursor was transformed into $\mathrm{SnO}_{2}$ nanofibers.

\section{Results and Discussions}

\subsection{SEM}

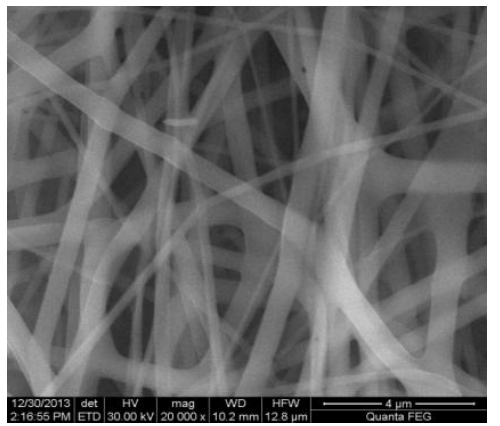

Figure 1.1: SEM images of Polyvinyl alcohol

The Scanning electron microscope was used to analyze the surface morphology of the fibers. Fig shows the SEM images of polyvinyl alcohol (PVA) and it confirms that PVA can be able to form the fibers at nanorange. It demonstrates that the smooth surface was formed. Fig. (a, b, c and d) show SEM images for a sample of Tin Oxide-PVA nanofibers at different magnification with a diameter of $450 \mathrm{~nm}$. Fig (a, b, $c$ and d) shoes the SEM images for a sample of $500^{\circ} \mathrm{C}$ annealed Tin Oxide nanofibers. It can be seen that the fbers are quite uniform with a diameter of about $250 \mathrm{~nm}$, and the porous structure can be observed as a result of the decomposition of PVA during annealing.

\subsection{Energy Dispersive X-Ray Spectroscopy:}

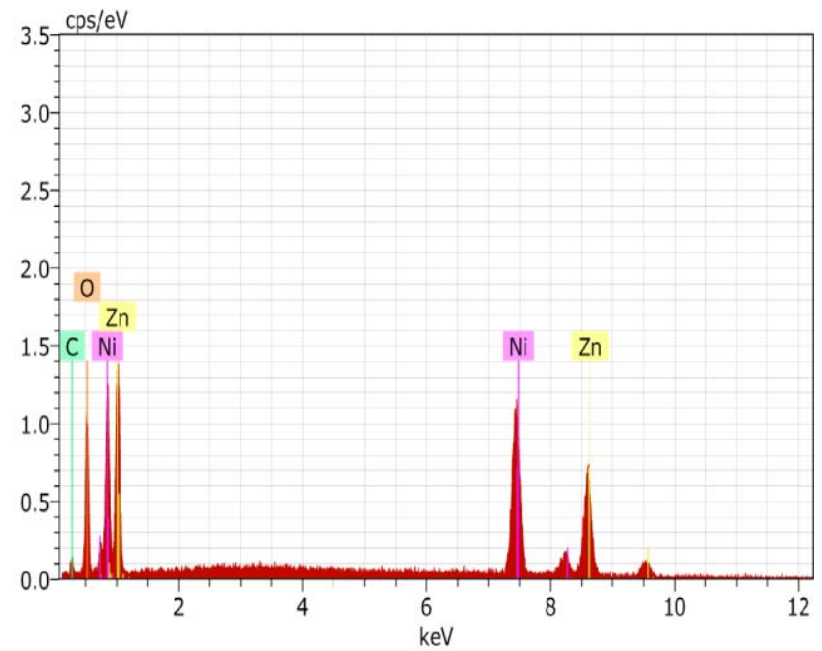

El All Series unn. C norm. C Atom. C Error (1 Sigma [wt. है] [ut. है] [at.ई] [ut. \$]

\begin{tabular}{|c|c|c|c|}
\hline 2 n $30 \mathrm{~K}$-series & 31.35 & 41.23 & 22.68 \\
\hline ii 28 R-series & 27.44 & 36.09 & 22.12 \\
\hline $8 \mathrm{R}$-series & 12.98 & 17.07 & 38.39 \\
\hline $6 \mathrm{~K}$-series & 4.27 & 5.61 & 16.81 \\
\hline
\end{tabular}

Total: $76.03 \quad 100.00 \quad 100.00$

Figure 1.2: EDX spectra of $500^{\circ} \mathrm{C}$ annealed tin oxide nanofibers

The EDX spectrum shown in Fig.6.4 indicates that the atomic ratio of Tin Oxide in the resultant Tin Oxide nanofibers is around $1: 1$, which is consistent with the composition of the precursor solution. This figure confirms the presence of the chemical composition in the sample.

\subsection{XRD:}

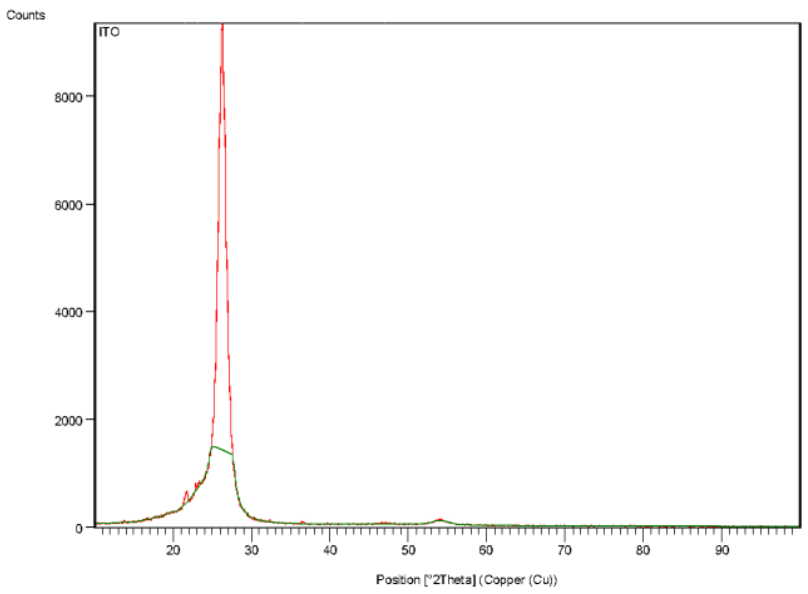

Figure 1.2: XRD image of as-spun PVA/Tin Oxidenanocomposite fibers

The XRD result for unannealed PVA composite fibers shows that the polymer count has larger than the composites. This is because that the ratio of polymer to the nanocomposite is $2: 1$. So, this result shows that it has less 


\section{International Journal of Science and Research (IJSR) \\ ISSN (Online): 2319-7064 \\ Index Copernicus Value (2013): 6.14 | Impact Factor (2014): 5.611}

count for the nanocomposite material. X-ray powder diffraction measurements were carried out to substantiate the structural and phase composition of the samples. It shows typical XRD patterns of the resultant Tin Oxide nanofibers. The main characteristic peaks of Tin Oxide from JCPDS card no. 65-2901 and SnO from JCPDS card no. 36-1451 are also given for comparison. The formation of cubic crystalline $\mathrm{SnO}$ in the hybrid is revealed by the diffraction peaks centered at $37.25^{\circ}, 43.35^{\circ}$ and $62.89^{\circ}$ corresponding to the (111), (200) and (220) crystal planes, respectively. The diffraction peaks at values of $31.72^{0}, 36.27^{\circ}$ and $62.75^{\circ}$ are ascribed to the wurtzite crystal structure of $\mathrm{SnO}$, which correspond to the (100), (101) and (110) crystal planes, respectively. From Fig a, The XRD measurement demonstrates that the hybrid is composed of crystalline $\mathrm{NiO}$ and $\mathrm{ZnO}$, which agrees with the results shown in Fig. . Furthermore, the diffraction peaks of $\mathrm{SnO}$ are sharp and intense, implying the high crystallinity of the nanofibers.

\subsection{UV-Visible Spectrometer}

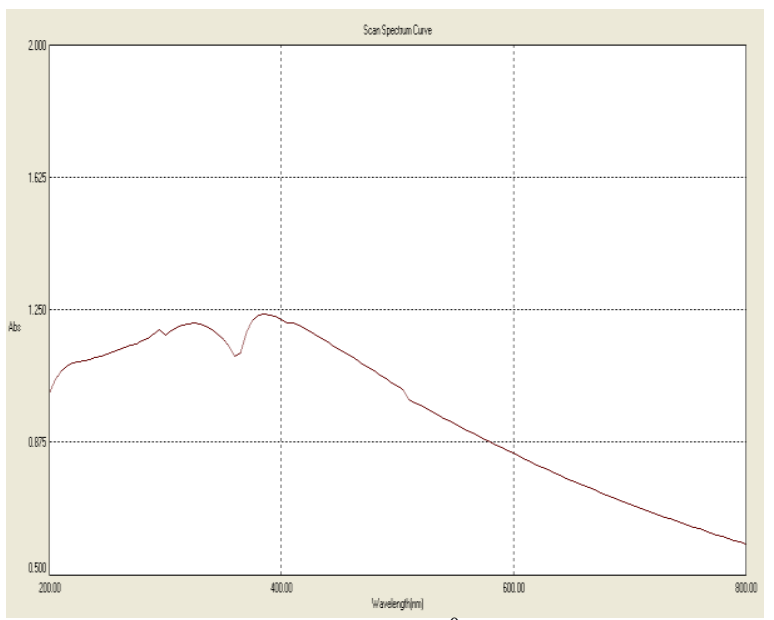

Figure 1.3: UV-VIS of $500^{\circ} \mathrm{C} \mathrm{SnO}$ nanofibers

\begin{tabular}{|c|c|c|}
\hline & Wavelength(nm) & Absorption \\
\hline 1 & 385 & 1.240 \\
\hline 2 & 325 & 1.213 \\
\hline
\end{tabular}

\subsection{Sensing Setup}

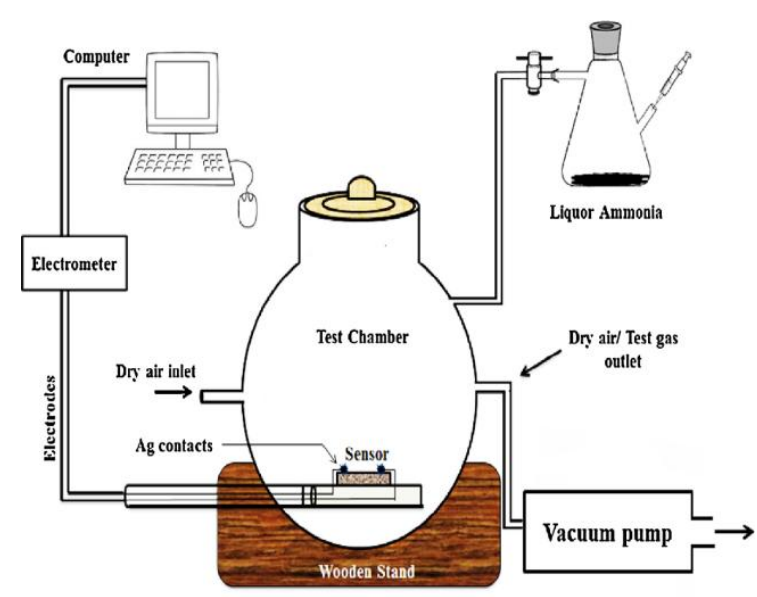

\subsubsection{Sensor Test}

Here fiber coated glass substrate is taken for sensor testing. The sample is dried at $60 \mathrm{C}$ for 2 hours and then taken for sensor testing. The volatile gases such as Formaldehyde (HCHO), Ammonia $\left(\mathrm{NH}_{3}\right)$ and Methane $\left(\mathrm{CH}_{4}\right)$ are used for testing the sample. First the silver paste is applied like a little drop on the sample and then heated in oven for $100^{\circ} \mathrm{CThe}$ sample is connected to the resistance meter via two copper wires through silver paste. The sample is kept in the atmospheric air to check the base resistance. Then the sample is kept inside the chamber and closed it. The target gas is allowed to pass inside the chamber. The resistance meter records the resistance value of the sample when target gas flows inside the chamber.

\subsubsection{Electro Meter/High Resistance Meter (6517 Hi-R METER)}

This high resistance meter is used to make -Current, Voltage and Resistance measurement values of sensing device.

\subsubsection{Sensitivity Formula}

\section{$\mathbf{S}=\mathbf{R}_{\mathrm{a}}-\mathbf{R}_{\mathrm{g}}$}

$\mathbf{R}_{\mathbf{a}}$ where $R_{a}-$ Resistance in air $\mathrm{R}_{\mathrm{g}}-$ Resistance in gas

\subsection{Sensing Gases}

\section{AMMONIA $\left(\mathrm{NH}_{3}\right)$ :}
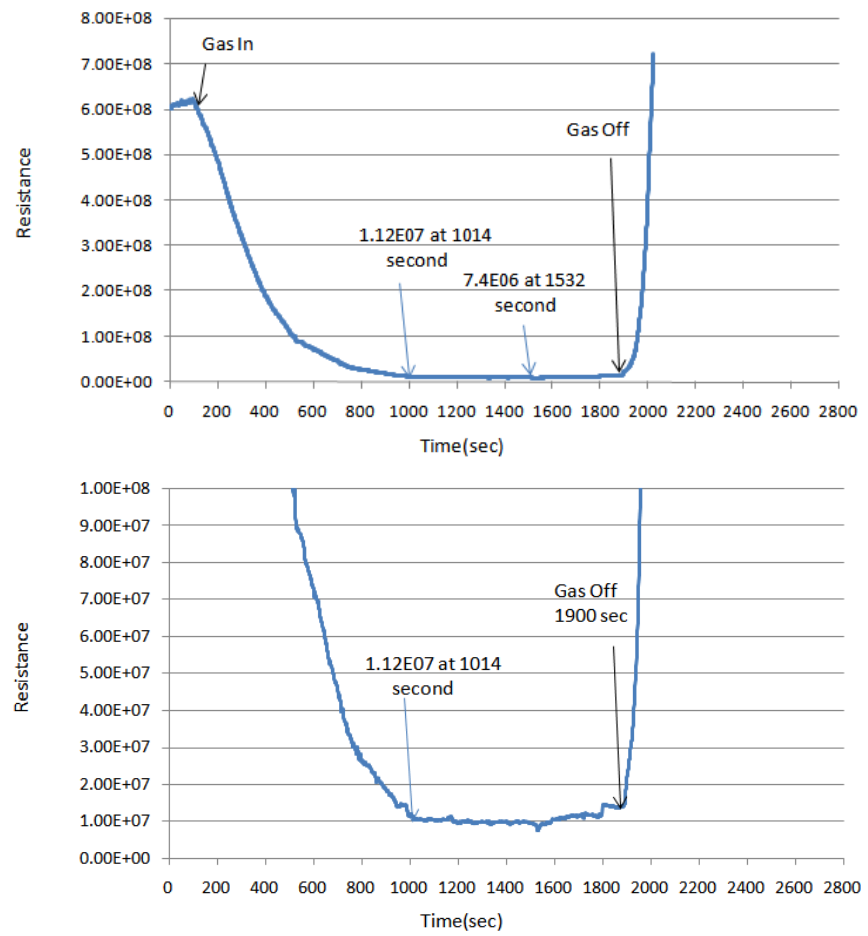

Figure 1.4: Resistance response of as-spun PVA-SnO nanofibers towards ammonia gas

\section{$2 \mathrm{NH}_{3}+5 \mathrm{O}^{-}(\mathrm{ad}) \rightarrow 2 \mathrm{NO}+3 \mathrm{H} 2 \mathrm{O}+5 \mathrm{e}^{-}$}

The base resistance value is $6.1 \mathrm{E} 8$. After the base resistance is taken, the gas is passed at $150^{\text {th }}$ second and the curve gradually decreases from E8 to E7 and gets constant after 1500 seconds. This indicates that the sample has sensitivity for ammonia gas. Finally the vacuum pump is switched ON to release the gases present inside the chamber and the 


\section{International Journal of Science and Research (IJSR) \\ ISSN (Online): 2319-7064}

Index Copernicus Value (2013): 6.14 | Impact Factor (2014): 5.611

resistance curve increases. PVA restricts some amount of gas sensitivity to the sample; still it shows good sensitivity of $98 \%$ for ammonia gas.

\section{Formaldehyde:}

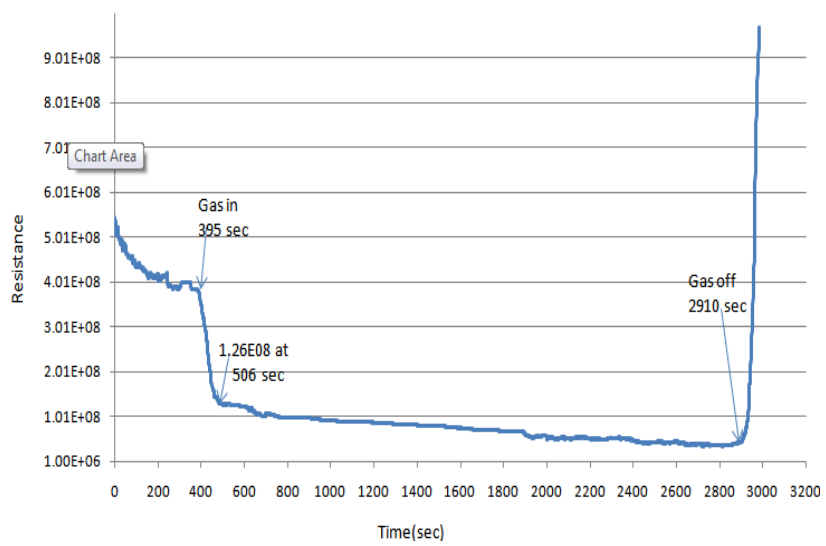

Figure 1.5: Resistance response of as-spun nanofibers towards formaldehyde gas

The above figure shows that the base (or atmospheric) resistance took for 400 seconds and the average base resistance value is $4.5 \mathrm{E} 8$. Then the chamber is opened and the formaldehyde (liquid form) is allowed to pass inside the chamber close to the sample and the chamber is closed. Then the resistance curve decreases when the gas passes to the sample. The sensor testing is continued for 2000 seconds. The curve decreases from E9 to E7 and then it became constant. It indicates that the sample senses the formaldehyde gas. Because of the presence of some amount of polymer in the sample, it restricts the gas sensing. Finally the vacuum pump is swiched $\mathrm{ON}$ to release the gases present inside the chamber and the curve increases. The sensitivity is $73 \%$

\section{Methane}

It shows the change in resistance value of the sample heated at $100^{\circ} \mathrm{C}$ for methane gas at different ppm. From 6.11 ( $a, b$, c and d), it shows different gas sensitivity. At3000 ppm, it shows poor sensing and has sensitivity of $0.87 \%$. At 4000 ppm, it has sensitivity of $1.7 \%$. For $5000 \mathrm{ppm}$, it has sensitivity of $2.4 \%$. At $6000 \mathrm{ppm}$, itshows good sensing and has $2.67 \%$ sensitivity. From this, we can understand that when the ppm level increases, sensitivity also increases.
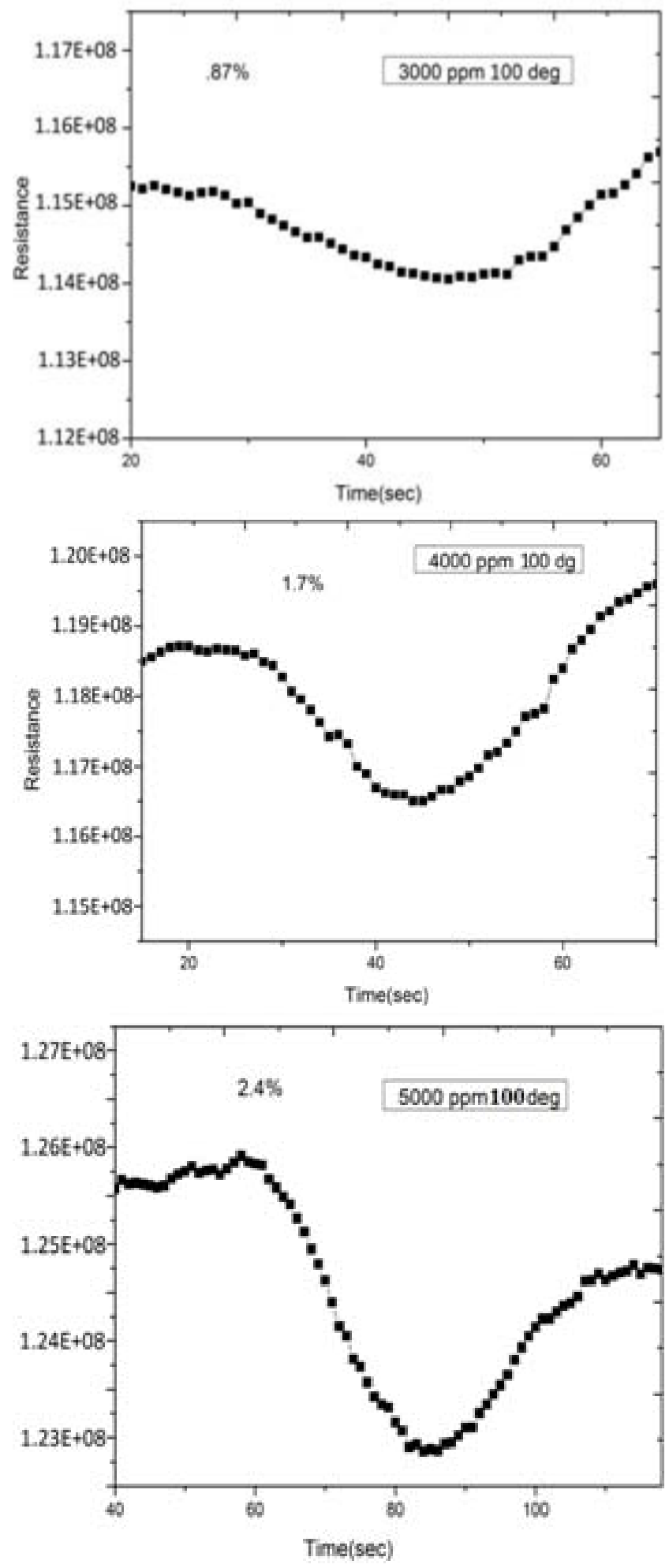

4. Conclusion

Successful deployment of chemosensors for breath testing would surely be cost-effective and highly beneficial for human health care, yet it involves many challenges. Discrimination between breath samples of diseased and healthy individual on the basis of trace level breath VOC differentiation demands high sensitivity and tunable selectivity. The demands are even further stiffened by real environmental conditions imposing unspecific interactions, humidity and temperature variations, and inhomogeneous test populations. Such a demanding framework compels an 


\section{International Journal of Science and Research (IJSR) \\ ISSN (Online): 2319-7064}

Index Copernicus Value (2013): 6.14 | Impact Factor (2014): 5.611

optimal choice of recognition approach and NMVSs for a given clinical state. For instance, in case clinical findings point toward a few specific breath markers with high BPs (indicating very low expression in breath), a specific recognition approach must be adopted employing highly sensitive nanomaterial transducers coupled to highly selective lockand-key receptors. When the indicative breath print involves a complex and uncertain combination of VOCs, a semiselective approach should be applied using arrays of crossreactive NMVSs. An array of NMVSs that combines both recognition approaches naturally performs an integration to yield a unique signal for complex but distinctive VOCs without requiring the mixture to be broken down into its individual components prior to, or during, the analysis. Future accurate breath testing systems that aim to detect diseases should incorporate a combination of technologies that amplify the signals originating from the breath markers, but also decrease the parasitic response originating from different confounding factors. The concentration of the relevant breath VOCs should be increased and humidity levels decreased through the use of microfabricated sample preparation devices, for instance, using a $\mu$-preconcentrator micro-GC. The processed sample will then be delivered to an array of NMVSs that will have an integrated on-chip temperature control unit. The type of recognition elements of the NMVS array would be tailored for maximum sensitivity and selectivity based on preevaluated breath markers. Following the trend of miniaturization in the world of technology, a breath testing system should eventually be able to fit into a casing as small as a smart-phone.

\section{References}

[1] Electrospinning of Nanomaterials and Applications in Electronic Components and Devices Jianjun Miao, Minoru Miyauchi, Trevor J. Simmons, Jonathan S. Dordick, and Robert J. Linhardt

[2] R. Jiri, P. Marek, V. Vladimir, Aligned nano fiber deposition onto a apatterened rotating drum collector by electrospinning, Brno, Czech Republic, EU, (2011)

[3] S. N. Reznik, , A. L. Yarin, , A. Theron, E. Zussman, Transient and steady shapes ofdroplets attached to a surface in a strong electric field. Journal of Fluid Mechanics, 516 (2004), 349-377.

[4] Ondarcuhu T, Joachim C. Drawing a single nanofibre over hundreds of microns. EurophysLett 1998;42(2):21520.

[5] Feng L, Li S, Li H, Zhai J, Song Y, Jiang L, et al. Super-Hydrophobic Surface of Aligned Polyacrylonitrile Nanofibers. Angew ChemInt Ed 2002;41(7):1221-3.

[6] Ma PX, Zhang R. Synthetic nano-scale fibrous extracellular matrix. J Biomed Mat Res 1999;46:60-72.

[7] Liu GJ, Ding JF, Qiao LJ, Guo A, Dymov BP, Gleeson JT, et al. Polystyrene-block-poly (2-cinnamoylethyl methacrylate) nanofibers-Preparation, characterization, and liquid crystalline properties. Chem-A European J 1999;5:2740-9.

[8] Deitzel JM, Kleinmeyer J, Hirvonen JK, BeckTNC. Controlled deposition of electrospun poly(ethylene oxide) fibers. Polymer 2001; 42:8163-70.
[9] Zheng-Ming Huanga, Y.-Z. Zhangb, M. Kotakic, S. Ramakrishna. A review on polymer nanofibers by electrospinning and their applications in nanocomposites. Composites Science and Technology 63 (2003) 2223-2253

[10] Jianjun Miao, Minoru Miyauchi4, Trevor J. Simmons, Jonathan S. Dordick, and Robert J. Linhardt. Electrospinning of Nanomaterials and Applications in Electronic Components and Devices. Journal of Nanoscience and Nanotechnology. Vol. 10, 5507-5519, 2010 